\title{
Compound Admittance Matrix Estimation of Three-phase Untransposed Power Distribution Grids using Synchrophasor Measurements
}

\author{
Rahul Kumar Gupta ${ }^{*, a}$, Fabrizio Sossan ${ }^{c}$, Jean-Yves Le Boudec ${ }^{b}$, Mario Paolone $^{a}$ \\ ${ }^{a}$ Distributed Electrical Systems Laboratory, EPFL, Switzerland, ${ }^{b}$ Laboratory for Communications and \\ Applications2, EPFL, Switzerland, ${ }^{c}$ PERSEE, Mines ParisTech - PSL, France.
}

\begin{abstract}
The paper considers the problem of estimating the parameters of the compound admittance matrix of three-phase untransposed low- and medium-voltage electrical distribution networks using synchrophasor measurements from phasor measurement units (PMUs). The work proposes and analyses the performance of a pre-processing strategy on the PMU's raw measurements which consists in grouping the raw measurements in clusters and, then, using the averaged measurements from each cluster for admittance matrix estimation. This step reduces the noise level and discards similar measurements from each cluster, ultimately improving the estimation quality of regressionbased estimation methods such as least squares (LS) and total least squares (TLS). The proposed approach uses a linear estimation model with phasor measurements of nodal voltages, nodal injection currents, and branch currents. We adopt a realistic measurement noise model in polar coordinates, which reflects the accuracy class of measuring instruments and is projected to rectangular coordinates. The proposed approach is validated by performing simulated experiments for different CIGRE and IEEE benchmark grids. Furthermore, the work includes different sensitivity analyses on the pre-processing policy (cluster type and size), availability of branch or injection currents measurements, as well as with different noise levels on the measurement data.
\end{abstract}

Index Terms-Admittance matrix, untransposed and unbalanced three-phase, least-squares, total least-squares, power distribution grids.

\section{INTRODUCTION}

The key management and protection functions for the power systems rely on the definition of suitable models that enable studying the behaviour of the grid under different operating conditions. Often, these models require accurate knowledge of the electrical parameters and the grid topology to estimate the state of the systems such as nodal voltages and branch currents [1]-[3]. These models are used for a number of applications such as optimal power flow (OPF)-based scheduling and control (e.g. [4], [5]), state estimation (e.g. [6]), locating faults (e.g. [7]), protection (e.g. [8]). Usually, these parameters are derived by either using the data-sheets from the components manufacturers [9] or by making off-line measurements [10]. The first method is widely applicable but the actual parameters can be quite different from the nominal ones due to various reasons, such as inaccurate information on the data-sheet, miscalibration or outdated parameters due to ageing (e.g. shunt capacitance of coaxial power cables). The second method is not practical as it requires non-negligible investments by the grid

*Corresponding author: rahul.gupta@epfl.ch. operator. Inaccurate information on the grid parameters often leads to erroneous modeling of the grid, causing inaccuracy in the estimates of grid analysis tools such as power-flow, stateestimation etc [11]-[14]. For example, [11] and [12] showed the effect of parameter errors on state estimation and power flows performance. The work in [14] shows the impact of parameter errors on differential protection. In this context, we propose a method to estimate the parameters of the compound admittance matrix of electrical distribution networks.

Thanks to the deployment of phasor measurement units (PMUs) and instrument transformers (ITs), the synchronized phasor measurements from these devices offer the possibility of data-driven estimation of the network parameters. At present, PMUs are largely deployed in the transmission grids, but they are also getting installed for monitoring and control of "active" distribution grids [15]-[17].

Recent works in this domain can be broadly clustered into three categories focusing on different aspects of the estimation process: $i$ ) assumption on the measurement noise model, ii) solution techniques used for the parameters estimation and iii) approximations in the modeling of the distribution grids.

The parameter estimation using PMUs measurements was initially proposed in [18], [19]. Thereafter, several works have been reported: the works in [20]-[24] proposed methods for estimation of line parameters in transmission networks. The work in [25] formulated the problem for identifying the admittance matrix directly from the synchronised measurements, but it did not account for a realistic noise model and the proposed model is very sensitive to even small noise. The work in [26] formulated the problem for joint-estimation of line parameters and topology using weighted total least squares (WTLS) method. The formulation is non-convex and is solved iteratively. The work assumes an unrealistic noise model by adding an offset noise from historical data, however lacking of physical significance. The works in [27]-[29] accounted for the systematic error of the ITs in the parameter estimation problem and used realistic measurement noise in polar coordinates. The work in [30] considered the noise in polar coordinates for three-phase systems and estimated equivalent error model for positive sequence components.

The use of least squares (LS) is proposed for estimating the parameters in [20]-[23], [25]. However, as shown in [31], it does not perform well when the measurements are corrupted with realistic measurement noise (in polar coordinates) as LS ignores noise in some variables. WLS based parameter 
estimation was used in [27]. Some works have proposed using error-in-variable (EIV) methods, such as the total least squares (TLS) [26]. The TLS solution is equivalent to the maximum likelihood estimation (MLE), when the noise on the input and output variables is approximated by white Gaussian distribution and is i.i.d. (independent and identically distributed), otherwise the estimations can be worse than LS, even if the TLS formulation is statistically more correct than the LS [31]. This happens because TLS tries to estimate not only the estimation variables but also the true and unobserved values of the measurements. Also, when TLS is fed with a large amount of measurements, its performance deteriorates due to large dimension of measurement to be de-noised. However, it is widely known that estimation methods achieve better performance when fed with inputs characterised by low measurement noise. For example, the work in [23] proposed a moving window averaging on the raw data for improving TLS based line parameter estimation by reducing the noise level. The method was proposed for estimation of a transposed and balanced line and used measurements of nodal powers along with currents and voltages. Although this work proposed an averaging strategy of raw PMU measurements, it did not fully use the fact that this step preserves the mathematical structure of the original formulation and improves the estimation performance.

Furthermore, in most of the existing works, it is often assumed that the power networks are balanced and transposed three-phase systems (e.g., [20]-[27]) with negligible shunt components. These assumptions do not hold at the same time for real distribution grids; the low voltage distribution networks are often untransposed [32], [33], and characterised by negligible shunt parameters, whereas medium voltage systems have non-negligible shunt parameters [32] (especially in the presence of long coaxial cables) and are relatively transposed systems. The works in [34]-[37] considered parameter estimation for a three-phase unbalanced and untransposed systems, but their analysis is limited to estimation of single line parameters instead of the whole compound admittance matrix and neglect shunt admittances. Estimating the whole compound admittance matrix is a complex problem as it requires to estimate all the branch parameters having different characteristics (e.g., short and long lines) within the network. Also these methods rely on measurements of line currents instead of injection currents which require twice the number of measuring instruments.

In this context, compared to the existing literature, the key contributions of this work are:

1) We propose a pre-processing strategy on the raw PMU measurements for improving the estimation performance of LS and TLS. The pre-processing consists of two main steps: first, the raw measurements are grouped into different clusters and, then, the averaged measurements from each cluster are used for admittance estimation. This process reduces the noise level of measurements, ultimately improving the estimation performance of LS and TLS.

2) We propose a PMU-based linear estimation model for estimating the whole admittance matrix for untransposed three-phase distribution networks (i.e. the so-called compound admittance matrix) taking into account both branch and shunt admittance matrices of the grid components (i.e. with no approximation on the grid model).

The estimation models adopt realistic noise in polar coordinates, reflecting the accuracy class of measuring instruments that is projected onto the rectangular coordinates. The method is validated by performing admittance estimation for different CIGRE and IEEE benchmark networks. Furthermore, we perform sensitivity analysis on the estimation performance with different noise levels on the measurement data, on the preprocessing strategy (cluster type and size), and availability of branch or injection currents measurements.

Compared to [20]-[23], [29], [34]-[37], the proposed method has the following features: $i$ ) it estimates the whole compound admittance matrix including the presence of shunt admittances, ii) it uses a pre-processing strategy for reducing the noise level ultimately improving the estimation performance, and iii) it works with either injection or branch/line current measurements, the former requires half the number of measurements. Compared to [25]-[27], the proposed method can be applied to untransposed three-phase grids accounting for the presence of shunt parameters. It is worth noting that this work does not assume knowing the nominal values of the branch and shunt parameters compared to [27], [28].

The paper is organized as follows: Section II presents the problem statement. Section III describes the estimation techniques and the proposed pre-processing strategy on the measurement data. Section IV introduces the case studies including the description of a realistic noise model for the measurements. Section V presents the estimation results and, finally, Section VI summarizes the main contributions.

\section{Problem Statement}

We consider the problem of estimating the parameters of the admittance matrix of a single- and three-phase distribution network (assuming that the topology is known) using a set of synchrophasor measurements that may include phase-toground nodal voltages, nodal injection currents and branch currents per phase.

In the following, we describe the poly-phase grid model, which is then used to formulate the parameter estimation problem for transposed and untransposed three-phase systems. We use the same nomenclature and hypothesis as in [38].

Consider the generic case of a ground-referenced ${ }^{1}$ unbalanced and untransposed ${ }^{2}$ polyphase power network with $\mathcal{G}:=\{0\}$ be the ground node, and $\mathcal{P}:=\{1, \ldots,|\mathcal{P}|\}$ the phases, $N$ buses and $L$ branches. The polyphase nodes, shunts and branch indices are collected in the sets $\mathcal{N}:=\{1, \ldots, N\}$, $\mathcal{T}:=\mathcal{N} \times \mathcal{G}=\mathcal{N}$ and $\mathcal{L}:=\{1, \ldots, L\}$ respectively. Figure 1 shows the topology of compound electrical parameters of a polyphase electrical circuit of the grid. The polyphase terminals and wires are bundled into a line for the

\footnotetext{
${ }^{1} \mathrm{We}$ assume that there exists a reference node i.e., ground, used as a reference to measure the nodal phase-to-ground voltages.

${ }^{2}$ Therefore, branch impedances and shunt admittance matrices are not necessarily circular-symmetric and triplets of three-phase voltages and currents are unsymmetrical and unbalanced.
} 
sake of clarity. The compound branch impedance and shunt admittance matrices are denoted by $\mathbf{Z}_{l}(l \in \mathcal{L})$ and $\mathbf{Y}_{t}(t \in \mathcal{T})$ respectively.

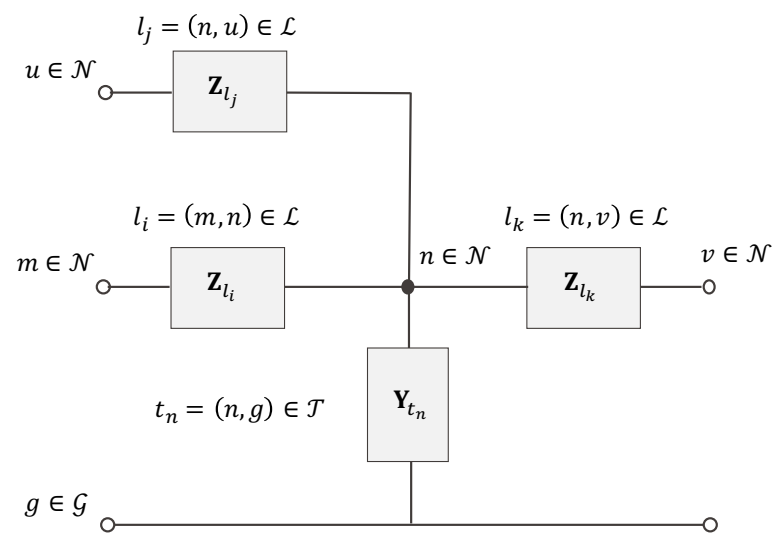

Fig. 1. Compound electrical parameters of the overall electrical circuit of the grid: compound branch impedance matrices $\mathbf{Z}_{l}(l \in \mathcal{L})$ and shunt admittance matrices $\mathbf{Y}_{t}(t \in \mathcal{T})$. The polyphase terminals and wires are bundled into single line for the sake of clarity.

Hypothesis 1: The grid consists of electrical components which are passive and linear. The coupling between the phases of the same component is significant, they can be represented by polyphase $\Pi$-section or T-section equivalent circuits.

Hypothesis 2: The compound branch impedance matrices are symmetric $\left(\mathbf{Z}_{l}=\mathbf{Z}_{l}^{T}\right)$, invertible $\left(\exists \mathbf{Y}_{l}=\mathbf{Z}_{l}^{-1}\right)$, and lossy $\left(\Re\left\{\mathbf{Z}_{l}\right\} \succcurlyeq 0\right), \forall l \in \mathcal{L}$. The compound shunt admittance matrix $\mathbf{Y}_{t}$ is also symmetric $\left(\mathbf{Y}_{t}=\mathbf{Y}_{t}^{T}\right)$, invertible $\left(\exists \mathbf{Z}_{t}=\mathbf{Y}_{t}^{-1}\right)$, we assume that the shunts are lossless $\left(\Re\left\{\mathbf{Y}_{t}\right\}=0\right), \forall t \in \mathcal{T}$.

It is worth pointing out that the symmetric property is a consequence of reciprocity of electromagnetism imposed by Maxwell's equation.

Let $V_{n, p}$ and $I_{n, p}$ be the phasors of the nodal phase-toground voltage and the injected current for the phase $p \in \mathcal{P}$ of the polyphase node $n \in \mathcal{N}$. The quantities defined for a polyphase node $n \in \mathcal{N}$ as a whole is written as:

$$
\begin{aligned}
& \mathbf{V}_{n}:=\operatorname{col}_{p \in \mathcal{P}}\left(V_{n, p}\right) \\
& \mathbf{I}_{n}:=\operatorname{col}_{p \in \mathcal{P}}\left(I_{n, p}\right)
\end{aligned}
$$

and for the grid as a whole is

$$
\begin{aligned}
& \mathbf{V}:=\operatorname{col}_{n \in \mathcal{N}}\left(\mathbf{V}_{n}\right) \\
& \mathbf{I}:=\operatorname{col}_{n \in \mathcal{N}}\left(\mathbf{I}_{n}\right)
\end{aligned}
$$

where the operator "col" constructs a column vector.

From Ohm's law, the injection currents is related to the nodal voltage as:

$$
\mathbf{I}=\mathbf{Y V},
$$

where $\mathbf{Y}$ is the compound admittance matrix defined as:

$$
\mathbf{Y}=\left(\mathbf{A}^{\mathcal{P}}\right)^{T} \mathbf{Y}_{\mathcal{L}} \mathbf{A}^{\mathcal{P}}+\mathbf{Y}_{\mathcal{T}}
$$

where,

$$
\begin{aligned}
& \mathbf{Y}_{\mathcal{L}}=\operatorname{diag}_{l \in \mathcal{L}}\left(\mathbf{Y}_{l}\right) \\
& \mathbf{Y}_{\mathcal{T}}=\operatorname{diag}_{t \in \mathcal{T}}\left(\mathbf{Y}_{t}\right)
\end{aligned}
$$

are the primitive compound branch and the primitive compound shunt admittance matrices respectively. The symbol $\mathbf{A}^{\mathcal{P}}$ denotes the polyphase incidence matrix and is defined as

$$
\mathbf{A}^{\mathcal{P}}=\mathbf{A} \otimes \operatorname{diag}\left(\mathbf{1}_{|\mathcal{P}|}\right)
$$

where $\left(\mathbf{1}_{|\mathcal{P}|}\right)$ is a vector of ones with length $|\mathcal{P}|, \otimes$ refers to the Kronecker product, and $\mathbf{A}$ is the incidence matrix obtained form the graph comprising of network branches as in [38].

Using (5) and (6), the injected current can be re-written as

$$
\begin{aligned}
& \mathbf{I}=\left(\left(\mathbf{A}^{\mathcal{P}}\right)^{T} \mathbf{Y}_{\mathcal{L}} \mathbf{A}^{\mathcal{P}}+\mathbf{Y}_{\mathcal{T}}\right) \mathbf{V} \\
& \mathbf{I}=\left(\mathbf{A}^{\mathcal{P}}\right)^{T} \mathbf{I}_{\mathcal{L}}+\mathbf{I}_{\mathcal{T}}
\end{aligned}
$$

where $\mathbf{I}_{\mathcal{L}}$ and $\mathbf{I}_{\mathcal{T}}$ refer to the branch and shunt current which are defined analogously as in (2) and (4).

In the following, we show how eqs. (1) - (11) can be used to derive linear models with branch and shunt parameters for transposed and untransposed distribution grids, which will be later used to estimate the compound admittance matrix.

1) Balanced and transposed three-phase case: A balanced and transposed system can be represented by a single-phase equivalent model, i.e $|\mathcal{P}|=1$ or $\mathbf{A}^{\mathcal{P}}=\mathbf{A}$. Using the definitions (7) and (8) and (10) the injected current can be re-written as

$$
\begin{array}{r}
\mathbf{I}=\left(\mathbf{A}^{T} \operatorname{diag}_{l \in \mathcal{L}}\left(\mathbf{Y}_{l}\right) \mathbf{A}+\operatorname{diag}_{t \in \mathcal{T}}\left(\mathbf{Y}_{t}\right)\right) \operatorname{col}_{n \in \mathcal{N}}\left(\mathbf{V}_{n}\right) \\
\text { or, } \mathbf{I}=\mathbf{A}^{T} \operatorname{diag}(\mathbf{A V}) \operatorname{col}_{l \in \mathcal{L}}\left(\mathbf{Y}_{l}\right) \\
+\operatorname{diag}_{n \in \mathcal{N}}\left(\mathbf{V}_{n}\right) \operatorname{col}_{t \in \mathcal{T}}\left(\mathbf{Y}_{t}\right) .
\end{array}
$$

Similarly, the branch current can be expressed as

$$
\mathbf{I}_{\mathcal{L}}=\operatorname{diag}(\mathbf{A V}) \operatorname{col}_{l \in \mathcal{L}}\left(\mathbf{Y}_{l}\right)
$$

Let us define the following matrices:

$$
\begin{aligned}
& \mathbf{C}=\operatorname{diag}(\mathbf{A} \Re\{\mathbf{V}\}) \\
& \mathbf{D}=\operatorname{diag}(\mathbf{A} \Im\{\mathbf{V}\}) \\
& \mathbf{E}=\operatorname{diag}(\Im\{\mathbf{V}\}) \\
& \mathbf{F}=\operatorname{diag}(\Re\{\mathbf{V}\}) \\
& \mathbf{X}^{\mathbf{G}}=\operatorname{col}_{l \in \mathcal{L}}\left(\Re\left\{\mathbf{Y}_{l}\right\}\right) \\
& \mathbf{X}^{\mathbf{B}}=\operatorname{col}_{l \in \mathcal{L}}\left(\Im\left\{\mathbf{Y}_{l}\right\}\right) \\
& \mathbf{X}^{\mathbf{T}}=\operatorname{col}_{t \in \mathcal{T}}\left(\Im\left\{\mathbf{Y}_{t}\right\}\right)
\end{aligned}
$$

then, the injected and branch current can be re-written as

$$
\begin{aligned}
& {\left[\begin{array}{c}
\Re\{\mathbf{I}\} \\
\Im\{\mathbf{I}\}
\end{array}\right]=\left[\begin{array}{ccc}
\mathbf{A}^{T} \mathbf{C} & -\mathbf{A}^{T} \mathbf{D} & -\mathbf{E} \\
\mathbf{A}^{T} \mathbf{D} & \mathbf{A}^{T} \mathbf{C} & \mathbf{F}
\end{array}\right]\left[\begin{array}{l}
\mathbf{X}^{\mathbf{G}} \\
\mathbf{X}^{\mathbf{B}} \\
\mathbf{X}^{\mathbf{T}}
\end{array}\right] \text {, and }} \\
& {\left[\begin{array}{l}
\Re\left\{\mathbf{I}_{\mathcal{L}}\right\} \\
\Im\left\{\mathbf{I}_{\mathcal{L}}\right\}
\end{array}\right]=\left[\begin{array}{cc}
\mathbf{C} & -\mathbf{D} \\
\mathbf{D} & \mathbf{C}
\end{array}\right]\left[\begin{array}{l}
\mathbf{X}^{\mathbf{G}} \\
\mathbf{X}^{\mathbf{B}}
\end{array}\right]}
\end{aligned}
$$

respectively. Here, $\Re($.$) and \Im($.$) refer to the real and imagi-$ nary part of a complex quantity. 
2) Unbalanced and untransposed three-phase case: In this case, $|\mathcal{P}|=3$, the line and shunt parameters are matrices of dimension $\mathbb{C}^{3 \times 3}$. To express the line and shunt parameters in vector form, we propose transformations $\Gamma$ and $\Omega$ given below.

The line and shunt admittance parameters, $\mathbf{Y}_{l}$ and $\mathbf{Y}_{t}$ are symmetric (Hypothesis 2), we want to represent them by unique elements in a vector form. Let $Y_{l}^{m n}(m, n=1,2,3)$ be the unique elements of $\mathbf{Y}_{l}$, we propose following transformation:

$$
\boldsymbol{\Omega}\left(\mathbf{Y}_{l}\right)=\boldsymbol{\Omega}\left(\left[\begin{array}{ccc}
Y_{l}^{11} & Y_{l}^{12} & Y_{l}^{13} \\
Y_{l}^{12} & Y_{l}^{22} & Y_{l}^{23} \\
Y_{l}^{13} & Y_{l}^{23} & Y_{l}^{33}
\end{array}\right]\right)=\left[\begin{array}{l}
Y_{l}^{11} \\
Y_{l}^{22} \\
Y_{l}^{33} \\
Y_{l}^{12} \\
Y_{l}^{23} \\
Y_{l}^{13}
\end{array}\right]
$$

The same can be written for the shunt admittance parameters. To be able to express the branch and shunt parameters in vector form, the voltages need to be transformed as following: $\gamma\left(\mathbf{V}_{n}\right)$ $=$

$$
\gamma\left(\left[\begin{array}{l}
V_{n, 1} \\
V_{n, 2} \\
V_{n, 3}
\end{array}\right]\right)=\left[\begin{array}{cccccc}
V_{n, 1} & 0 & 0 & V_{n, 2} & 0 & V_{n, 3} \\
0 & V_{n, 2} & 0 & V_{n, 1} & V_{n, 3} & 0 \\
0 & 0 & V_{n, 3} & 0 & V_{n, 2} & V_{n, 1}
\end{array}\right]
$$

Similarly, this transformation is applied to all the nodes by $\boldsymbol{\Gamma}(\mathbf{V})=\operatorname{col}_{n \in \mathcal{N}}\left(\gamma\left(\mathbf{V}_{n}\right)\right)$.

Using the above transformation on all phase voltages and branch and shunt parameters, eqs. (16) and (17) can be written for a three-phase unbalanced and untransposed system with the following definitions:

$$
\begin{aligned}
& \mathbf{C}=\operatorname{diag}_{l \in \mathcal{L}}\left(\mathbf{A}_{l}^{\mathcal{P}} \Re\{\boldsymbol{\Gamma}(\mathbf{V})\}\right) \\
& \mathbf{D}=\operatorname{diag}_{l \in \mathcal{L}}\left(\mathbf{A}_{l}^{\mathcal{P}} \Im\{\boldsymbol{\Gamma}(\mathbf{V})\}\right) \\
& \mathbf{E}=\operatorname{diag}_{n \in \mathcal{N}}\left(\Im\left\{\gamma\left(\mathbf{V}_{n}\right)\right\}\right) \\
& \mathbf{F}=\operatorname{diag}_{n \in \mathcal{N}}\left(\Re\left\{\gamma\left(\mathbf{V}_{n}\right)\right\}\right) \\
& \mathbf{X}^{\mathbf{G}}=\operatorname{col}_{l \in \mathcal{L}}\left(\Re\left\{\boldsymbol{\Omega}\left(\mathbf{Y}_{l}\right)\right\}\right) \\
& \mathbf{X}^{\mathbf{B}}=\operatorname{col}_{l \in \mathcal{L}}\left(\Im\left\{\boldsymbol{\Omega}\left(\mathbf{Y}_{l}\right)\right\}\right) \\
& \mathbf{X}^{\mathbf{T}}=\operatorname{col}_{t \in \mathcal{T}}\left(\Im\left\{\boldsymbol{\Omega}\left(\mathbf{Y}_{t}\right)\right\}\right) \text {. }
\end{aligned}
$$

Here, $\mathbf{A}_{l}^{\mathcal{P}}$ is incidence matrix for $l$-th line, i.e. rows $((l-$ $1)|\mathcal{P}|+1)$ to $l|\mathcal{P}|$ in $\mathbf{A}^{\mathcal{P}}$.

The problem considered in this paper is as follows. We assume that the incidence matrix $\mathbf{A}^{\mathcal{P}}$ is known, and we have a collection of complex current $\mathbf{I}$ and voltage $\mathbf{V}$ measurements, from which the matrices $\mathbf{C}, \mathbf{D}, \mathbf{E}$ and $\mathbf{F}$ are immediately derived. The problem is then to estimate the unknown line and shunt parameters $\mathbf{X}^{\mathbf{G}}, \mathbf{X}^{\mathbf{B}}$ and $\mathbf{X}^{\mathbf{T}}$ by regression techniques in (16) and (17).

\section{METHODS}

Hypothesis 3: The line series impedances and shunt admittances are assumed to be constant during the period when measurements were collected. The change in these parameters due to temperature variations is neglected.

Hypothesis 4: We assume that the voltage and current phasor measurements are available at every node of the grid.
Using equations (16)-(17) and Hypotheses 3 and $\mathbf{4}$, the estimation problem can be formulated and solved by using standard estimation techniques such as least squares (LS) and total least squares (TLS). Eq. (16), in case of null measurement noise, can be written for a time-index $k$ as:

$$
\mathbf{J}(k)=\mathbf{H}(k) \mathbf{X}
$$

where $\mathbf{H}(k)=\left[\begin{array}{ccc}\mathbf{A}^{T} \mathbf{C}(k) & -\mathbf{A}^{T} \mathbf{D}(k) & -\mathbf{E}(k) \\ \mathbf{A}^{T} \mathbf{D}(k) & \mathbf{A}^{T} \mathbf{C}(k) & \mathbf{F}(k)\end{array}\right], \mathbf{J}(k)=$ $\left[\begin{array}{l}\Re\{\mathbf{I}(k)\} \\ \Im\{\mathbf{I}(k)\}\end{array}\right]$, and $\mathbf{X}=\left[\begin{array}{l}\mathbf{X}^{\mathbf{G}} \\ \mathbf{X}^{\mathbf{B}} \\ \mathbf{X}^{\mathbf{T}}\end{array}\right]$.

If we have $K$ measurements of voltage and current phasors, (21) can be expressed for all the $K$ data-points to obtain an over-determined system of linear equations as,

$$
\mathbf{J}=\mathbf{H X}
$$

where $\mathbf{J}=[\mathbf{J}(1) ; \ldots ; \mathbf{J}(K)]$ and $\mathbf{H}=[\mathbf{H}(1) ; \ldots ; \mathbf{H}(K)]$.

The equality in (22) holds in case of null noise, which is not the case for real measurements. In presence of the measurement noise, the observations $\mathbf{J}$ and $\mathbf{H}$ are given as:

$$
\begin{array}{ll}
\mathbf{J}=\widehat{\mathbf{J}}+\Delta \mathbf{J}, & \Delta \mathbf{J} \sim \mathcal{N}\left(0, \mathbf{Q}_{\mathbf{J}}\right) \\
\mathbf{H}=\widehat{\mathbf{H}}+\Delta \mathbf{H}, & \Delta \mathbf{H} \sim \mathcal{N}\left(0, \mathbf{Q}_{\mathbf{H}}\right)
\end{array}
$$

where, $\widehat{\mathbf{J}}$ and $\widehat{\mathbf{H}}$ are the true (unobserved) values, $\Delta \mathbf{J}$ and $\Delta \mathbf{H}$ are the measurement noises, and $\mathbf{Q}_{\mathbf{J}}$ and $\mathbf{Q}_{\mathbf{H}}$ represent the noise covariance matrices for $\mathbf{J}$ and $\mathbf{H}$ respectively.

It should be noted that the measurements on the branch currents (if available) can also be embedded in the estimation model using Eq. (17) in a similar way. In eq. (22), $\mathbf{X}$ is the unknown parameter to be estimated, whereas $\mathbf{H}$ and $\mathbf{J}$ are obtained using the voltage and current measurements from PMUs. In the following section, we describe the estimation techniques to estimate $\mathbf{X}$ in (22).

\section{A. Estimation techniques}

In the followings, we describe two estimation techniques which are used later to estimate the network parameters.

1) Least Squares (LS): the LS method assumes that noise $\Delta \mathbf{H}$ is negligible and the noise $\Delta \mathbf{J}$ is homoscedastic (i.e. elements of $\operatorname{diag}\left(\mathbf{Q}_{\mathbf{J}}\right)$ are all equal). The LS solution is:

$$
\mathbf{X}_{L S}^{*}=\left(\mathbf{H}^{T} \mathbf{H}\right)^{-1} \mathbf{H}^{T} \mathbf{J}
$$

2) Total Least Squares (TLS): in real measurements, $\mathbf{H}$ contains measurement noise, then LS solution is no longer optimal statistically. In [31], TLS was developed, which tries to estimate the true (unobserved) $\widehat{\mathbf{H}}$ and $\widehat{\mathbf{J}}$ along with $\mathbf{X}$. This leads to a non-linear and non-convex problem and has too many unknowns to determine. The work in [31] derived analytical solution, with an assumption that noise terms $\Delta \mathbf{J}, \Delta \mathbf{H}$ are Gaussian and i.i.d., using singular value decomposition (SVD) and is briefly described below.

Let the SVD of $[\mathbf{H} \mid \mathbf{J}]$ is

$$
[\mathbf{H} \mid \mathbf{J}]=\mathcal{U} \Sigma \mathcal{V}^{T}
$$


where, $\mathcal{V}=\left[\begin{array}{ll}\mathcal{V}_{\mathbf{H}, \mathbf{H}} & \mathcal{V}_{\mathbf{H}, \mathbf{J}} \\ \mathcal{V}_{\mathbf{J}, \mathbf{H}} & \mathcal{V}_{\mathbf{J}, \mathbf{J}}\end{array}\right], \Sigma=\operatorname{diag}(\bar{\sigma}, \ldots, \underline{\sigma})$.

The TLS solution is given as:

$$
\mathbf{X}_{T L S}^{*}=-\mathcal{V}_{\mathbf{H}, \mathbf{J}} \mathcal{V}_{\mathbf{J}, \mathbf{J}}^{-1} .
$$

Eq. (28) can be simplified when $\mathbf{J}$ is vector, it is given as

$$
\mathbf{X}_{T L S}^{*}=\left(\mathbf{H}^{T} \mathbf{H}-\underline{\sigma}^{2} I\right)^{-1} \mathbf{H}^{T} \mathbf{J}
$$

where, $\underline{\sigma}$ refers to the smallest singular value of $[\mathbf{H} \mid \mathbf{J}][39]$, [40]. Eq. (29) can be interpreted as method to remove the error co-variance matrix (approximated by $\underline{\sigma}^{2} I$ ).

By nature, when the TLS is fed with large number of measurements (with non-negligible noise), it attempts to estimate large amount of variables, namely the true value of the measurements and admittance parameters. This results in poor estimates as reported in [31]. To tackle this issue, we propose a pre-processing strategy on the raw measurements: a linear transformation that reduces the dimension of the input measurements as well as the noise level while preserving the original structure of the problem. Due to this pre-processing, the TLS is now trying to estimate the average values of the measurements. This task is easier than the original because there are fewer data points and reduced noise. The preprocessing strategy is described as follows.

\section{B. Measurement pre-processing by cluster averaging}

Measurement noise of PMUs and sensing instruments impacts $\mathbf{H}$ and $\mathbf{J}$ observation matrices. Realistic instrument accuracy classes (e.g., 0.5 and 1) lead to poor estimation performance, further aggravated by the nonlinear transposition from polar to rectangular coordinates, as reported in [37]. We propose to pre-process the input measurements because, as it will be shown later, it is conducive to improve the estimation performance. The pre-processing strategy consists in, first, grouping the raw measurements in a given number of clusters according to similarity features. Then, measurements within the same cluster are averaged and used in the estimation process instead of the raw measurements. The considered similarity features are: nodal voltage magnitudes, current magnitudes, and, for unbalanced and untransposed networks, sequence-domain voltage magnitudes and sequence-domain current magnitudes. The first group of features helps to detect different operating conditions in the grids due to large power variations and transient events (e.g., inrush currents), whereas the second, is more suitable for unbalanced conditions. The use of combinations of these features is discussed in the results section. Clustering and averaging the measurements achieve not only filtering out measurement noise but also reducing the number of similar measurements in the estimation process, improving the condition number of $\mathbf{H}$ and estimation performance of LS and TLS. It is worth highlighting that since averaging is linear, it can be efficiently implemented by applying a linear transformation to Eq. (22).

The cluster-averaging procedure is illustrated Algorithm 1. The kmeans algorithm is used for clustering. Inputs to the kmeans are the raw voltage and current phasor measurements $\{\mathbf{V}, \mathbf{I}\}$, features (defined above) and number of clusters $N_{c}$, and the outputs are voltage and current phasor measurements grouped in different clusters $\left[\{\mathbf{V}, \mathbf{I}\}_{1}, \ldots,\{\mathbf{V}, \mathbf{I}\}_{N_{c}}\right]$. After creating the clusters, the algebraic mean is computed for all the elements in the same cluster. We decided to use the kmeans as a clustering scheme as it is a widely used and effective method in unsupervised learning applications. Specifically, we used MATLAB built-in function that, regarding the clustering initialisation, uses the kmeans++ algorithm [41].

The similarity features, such as the magnitudes of the nodal voltages and currents, are used into the kmeans algorithm to decide the basis of the grouping of the raw measurements. We choose magnitudes of the voltages and currents as clustering features because $i$ ) as illustrated in [42], [43], the distribution of measurement noise is truly white in polar coordinates, $i$ ) magnitudes work well to identify the voltage and current imbalances in three-phase unbalanced systems and iii) it results in a linear estimation model, which is preserved by the averaging. Sec. V-D shows how the cluster-averaging process affects the grouping of the raw-measurements and their characteristics.

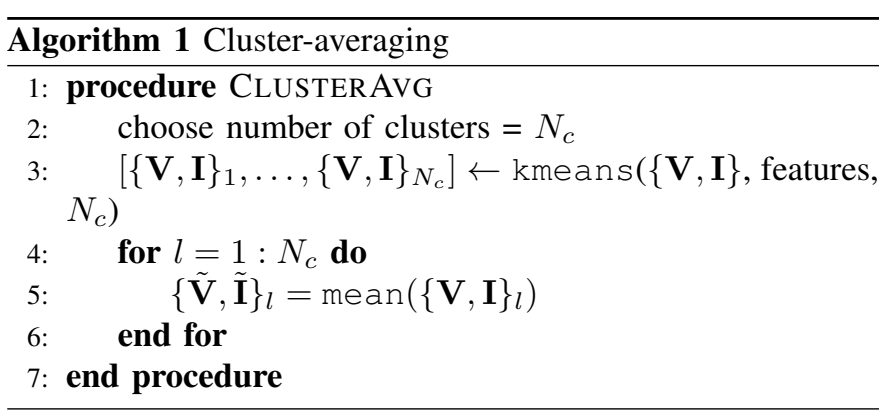

A naive averaging approach would be to perform block time-averaging of the measurements (Algorithm 2). However, this naive method has the disadvantage of losing important information associated to transients and unbalances contained in the raw measurements by averaging blindly time-contiguous data blocks. The advantage of cluster-averaging is that it groups and averages the data based on the similarity features and is independent of time. In the results section, we compare the estimation performance of the cluster-averaging $v s$ the block-averaging method.

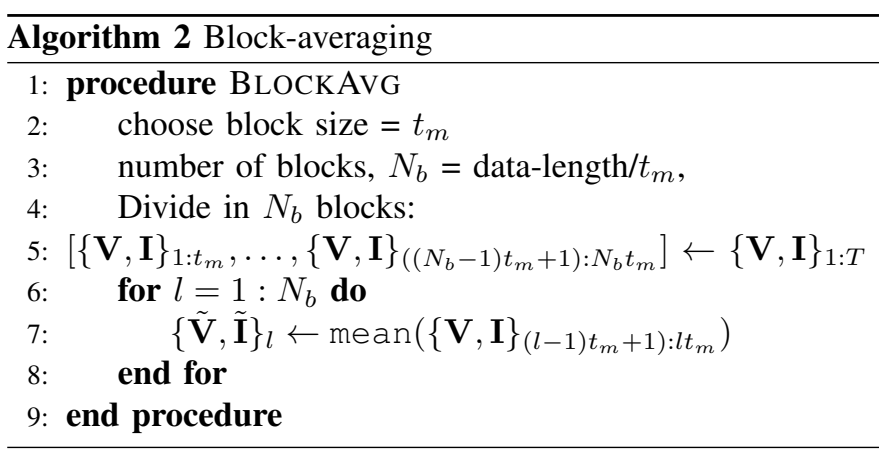

In summary, the measurement pre-processing is to obtain a proper grouping of the raw measurements. After creating the clusters, the algebraic mean is computed for all the elements (both the magnitudes and phases of the voltages and currents) in the same cluster. The averaged values (both the magnitudes and phases) are then used in the admittance matrix estimation. 


\section{Simulation SetuP}

\section{A. Input data-set}

To evaluate and compare the performance of the proposed method, we estimate the admittance parameters of selected power grid benchmarks starting from noisy phasor measurements, computed as follows. First, load flows are solved to compute ground-truth values of voltage and current phasors. Then, these are corrupted with i.i.d. zero-mean Gaussian noise to simulate noisy measurements, as discussed in the next subsection. This procedure is described in Algorithm 3. The nodal active and reactive power injections in the load flows are from the experimental set-up of the Distributed Electrical Systems Laboratory at EPFL [44] (peak consumption of $350 \mathrm{~kW}$ with $95 \mathrm{kWp}$ of PV generation). These measurements are rescaled according to the nominal power of the respective node of the test network. The rescaling is performed by first dividing the nodal demand/generation profiles of the original system in [44] by their nominal nodal powers and, then, multiplying by the nominal nodal powers of the test network.

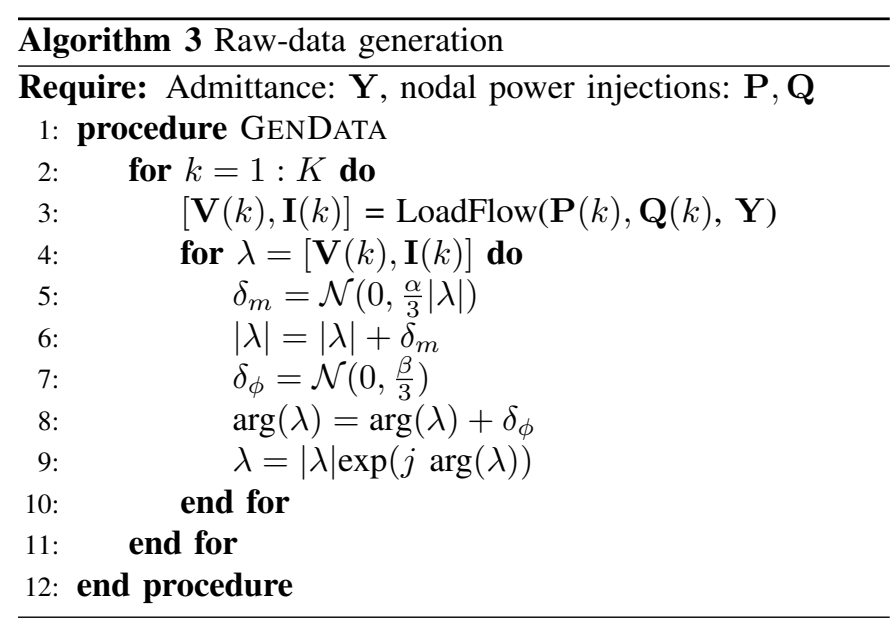

\section{B. Noise model}

The measurements of ITs are characterised by errors in polar coordinates, not in rectangular coordinates, therefore, we introduce noise in polar coordinates defined by the IT class types. The voltage and current measuring instruments are characterized by the phase and magnitude error, specified by the manufactures in form of percentage, $\alpha$, for magnitudes and in radians, $\beta$, for the phase error. The values of $\alpha$ and $\beta$ are listed in the Table I as defined by [42], [43].

TABLE I

ERRORS SPECIFICATIONS FOR DIFFERENT CLASS OF INSTRUMENT TRANSFORMERS DEFINED BY [42], [43].

\begin{tabular}{|c|c|c|c|c|}
\hline IT class & \multicolumn{2}{|c|}{ Voltage transformers } & \multicolumn{2}{c|}{ Current transformers } \\
\hline & $\begin{array}{c}\text { mag. error } \\
(\boldsymbol{\alpha})[\%]\end{array}$ & $\begin{array}{c}\text { phase error } \\
\boldsymbol{\beta}[\mathbf{r a d} .]\end{array}$ & $\begin{array}{c}\text { mag. error } \\
(\boldsymbol{\alpha})[\%]\end{array}$ & $\begin{array}{c}\text { phase error } \\
\boldsymbol{\beta}[\text { rad.] }\end{array}$ \\
\hline 0.1 & 0.1 & $1.5 \mathrm{e}-3$ & 0.1 & $1.5 \mathrm{e}-3$ \\
0.2 & 0.2 & $3 \mathrm{e}-3$ & 0.2 & $3 \mathrm{e}-3$ \\
0.5 & 0.5 & $6 \mathrm{e}-3$ & 0.5 & $9 \mathrm{e}-3$ \\
1 & 1 & $12 \mathrm{e}-3$ & 1 & $18 \mathrm{e}-3$ \\
\hline
\end{tabular}

Hypothesis 5: The ITs do not have a bias, and they behave according to standards; the magnitude and phase angle error of the ITs are Gaussian. The noise introduced by the PMUs is negligible compared to the one of the ITs (e.g. [45], [46]).

We add a Gaussian and i.i.d. unbiased noise (Hypothesis 5) to the voltage and current measurements in the polar coordinates, which is then projected onto the rectangular coordinates. Although Gaussian property may not be preserved upon the transformation, the transformed noise can be approximated to a Gaussian distribution for the IT classes ${ }^{3}$ of Table I as shown in [37].

\section{Performance metrics}

We measure the performance of the estimation algorithm by the three following metrics.

- The Normalized Mean Square Error (NMSE) between the true $(\widehat{\mathbf{X}})$ and the estimated $\left(\mathbf{X}^{*}\right)$ quantities as:

$$
\mathcal{E}\left(\mathbf{X}^{*}, \widehat{\mathbf{X}}\right)=\mathcal{E}_{\mathbf{X}}=\frac{\left\|\widehat{\mathbf{X}}-\mathbf{X}^{*}\right\|_{2}}{\|\widehat{\mathbf{X}}\|_{2}}
$$

$\mathcal{E}_{\mathbf{X}}$ is a dimensionless quantity, the value $\mathcal{E}_{\mathbf{X}}=0$ indicates a perfect estimation.

- Comparison in terms of per unit (pu) of the estimated and original parameters; pu is computed by dividing the parameter impedance/admittance by the base impedance/admittance. The base impedance/admittance is computed using the base power and base voltage of the system.

- Element-wise relative error of the estimated admittance. It is expressed for real and imaginary parts of each element of the compound admittance matrix defined as

$$
\Delta \mathbf{Y}=\widehat{\mathbf{Y}}-\mathbf{Y}^{*}=\Re(\Delta \mathbf{Y})+j \Im(\Delta \mathbf{Y})
$$

where $\widehat{\mathbf{Y}}$ and $\mathbf{Y}^{*}$ are true and estimated admittance matrices, $\Delta \mathbf{Y}$ is the element-wise error on estimated admittance. We define the relative element-wise error of estimated admittance on real $\left(\Delta_{\Re} \tilde{\mathbf{Y}}\right)$ and imaginary $\left(\Delta_{\Im} \tilde{\mathbf{Y}}\right)$ elements as

$$
\begin{aligned}
& \Delta_{\Re} \tilde{\mathbf{Y}}=\Re(\Delta \mathbf{Y}) \oslash \Re(\widehat{\mathbf{Y}}) \\
& \Delta_{\Im} \tilde{\mathbf{Y}}=\Im(\Delta \mathbf{Y}) \oslash \Im(\widehat{\mathbf{Y}})
\end{aligned}
$$

where $\oslash$ refers to Hadamard division (i.e., elementwise division of a matrix). Later, we show these relative component-wise errors on the real and imaginary part of the admittance matrix in a heatmap plots for the different strategies. $\Delta_{\Re} \tilde{\mathbf{Y}}, \Delta_{\Im} \tilde{\mathbf{Y}}$ are dimensionless as they are relative errors (these relative errors are zero for a perfect estimation).

- Uncertainty of the estimated parameters: is computed by their variances. The variances of the estimates are given by

$$
\left.\sigma_{\mathbf{X}}=\sqrt{(} \sigma_{r}^{2} \operatorname{diag}\left(\mathbf{H}^{T} \mathbf{H}\right)^{-1}\right)
$$

${ }^{3}$ According to [42], [43], the maximum measurement noise for a calibrated IT (i.e. without a bias) is associated to the the magnitude and phase errors reported in Table I. 


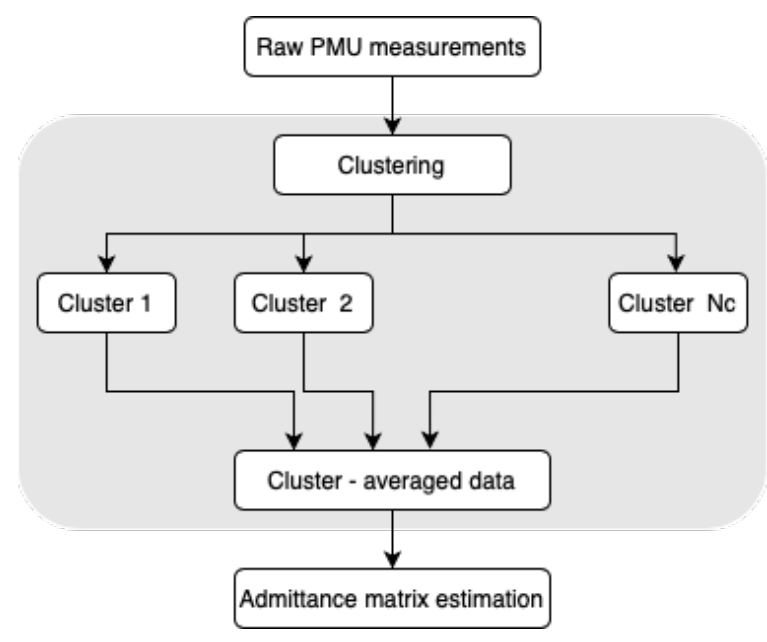

Fig. 2. Flow diagram for the admittance matrix estimation using clusteraveraged data.

where $\sigma_{r}$ is estimated variance of the residuals in (22) post-estimation. We determine the uncertainty corresponding to $99 \%$ confidence interval approximated by $\pm 3 \sigma_{\mathbf{X}}$.

\section{Results And Discussion}

We use the data sets from simulated experiments on different benchmark distribution grids, as described earlier, to estimate their admittance parameters. We compare the estimation performances using: $i$ ) the raw measurements (denoted by "Raw-data"), ii) the pre-processed data with block-averaging (denoted by "Block-averaging") and iii) the pre-processed data with cluster-averaging (denoted by "Cluster-averaging"). Figure 2 summarizes the steps used for the estimation using cluster-averaged data. Raw measurements are directly used for the estimations in the "Raw-data" case. They are not subject to any pre-processing and all the measurements are used once for the estimation of the parameters. The results are reported for both LS and TLS techniques.

A sensitivity analysis w.r.t different levels of measurement noise is presented. Then, a performance comparison is presented against different clustering strategy (such as cluster features, number of clusters). And finally, we compare the estimation performances with availability of branch and injection currents measurements.

\section{A. Estimation performance on benchmark test cases}

We present a detailed estimation performance analysis applied to the IEEE 4-bus benchmark network. It is an unbalanced and untransposed three-phase distribution system with artificially added shunt parameters. The details on the parameters, topologies and nominal demand can be found in [33]. The topology is shown in Fig. 3. The estimations are performed with an estimation model comprising of both the injection and branch currents measurements. We use a single day of simulated measurements of nodal voltages, branch, and injection currents (Algorithm 3) at a 1-second resolution. We first consider instrument type of class 0.2 (Table I). We

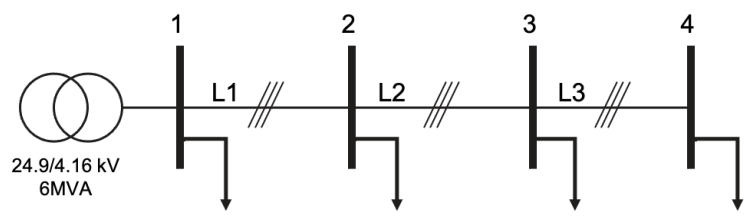

Fig. 3. Topology of the adopted IEEE 4-bus network.

compare the estimation performance when using directly the raw measurements (from Algorithm 3), block-averaging (first Algorithm 3 then Algorithm 2) and cluster-averaging (first Algorithm 3 then Algorithm 1). For the first one, we use all the measurements for the admittance estimation. For the last two, the estimations are compared using the same number of blocks as the number of clusters to have a fair comparison. We use 1-hour blocks in the block-averaging method of Algorithm 2 and 24 clusters (24 clusters in a day according to blockaveraging of 1-hour as blocks) in the cluster-averaging method of Algorithm 1. The corresponding averaged values of each cluster or block are used for the admittance matrix estimation. A sensitivity with cluster size and features will be discussed later in the paper.

Table II shows the estimation performance for IEEE 4-bus unbalanced and untransposed three-phase network. It shows

TABLE II

ESTIMATION PERFORMANCE FOR THE IEEE 4-BUS NETWORK.

\begin{tabular}{|c|c|c|c|c|c|}
\hline Data & Method & $\mathcal{E}_{\mathbf{X G}}$ & $\mathcal{E}_{\mathbf{X}}$ B & $\mathcal{E}_{\mathbf{X}} \mathbf{T}$ & $\mathcal{E}_{\mathbf{Y}}$ \\
\hline Raw-data & LS & 0.124 & 0.172 & 0.337 & 0.181 \\
& TLS & 0.027 & 0.035 & 0.147 & 0.038 \\
\hline Block- & LS & 0.191 & 0.145 & 0.016 & 0.163 \\
averaging & TLS & 0.229 & 0.117 & 0.021 & 0.137 \\
(1-hour block) & & & & & \\
\hline Cluster- & LS & 0.008 & 0.002 & 0.014 & 0.003 \\
averaging & TLS & 0.008 & 0.002 & 0.014 & 0.003 \\
(24 clusters) & & & & & \\
\hline
\end{tabular}

the NMSEs using LS and TLS techniques. The first, second and the third rows show estimations when using raw, blockaveraged and cluster-averaged data, respectively. It shows the NMSEs for conductances, susceptances, shunts and compound admittance matrices denoted by $\mathcal{E}_{\mathrm{X}^{\mathrm{G}}}, \mathcal{E}_{\mathrm{X}^{\mathrm{B}}}, \mathcal{E}_{\mathrm{X}^{\mathrm{T}}}$ and $\mathcal{E}_{\mathbf{Y}}$ respectively (as defined in (30)).

As it can be seen that the estimations are poor using the raw data, whereas they improve significantly with cluster-averaged data and thus have better estimation of the admittance matrix. With the cluster-averaging policy, the NMSEs decreases by 2-3 orders of magnitude compared to the raw-data. The estimations using block-averaging are also poor compared to the clusteraveraging and slightly better than the raw-data case. The block-averaging method performs the worst with TLS.

Fig. 4 shows the plots of the true and estimated threephase line and shunt parameters for the LS using the raw-data, block-averaging, and cluster-averaging cases. We also show the uncertainty of the estimates using error bars corresponding to $99 \%$ confidence interval. The upper, middle and the bottom plots show the comparison for the true and estimated conductances, susceptances and shunts, respectively. The estimates 


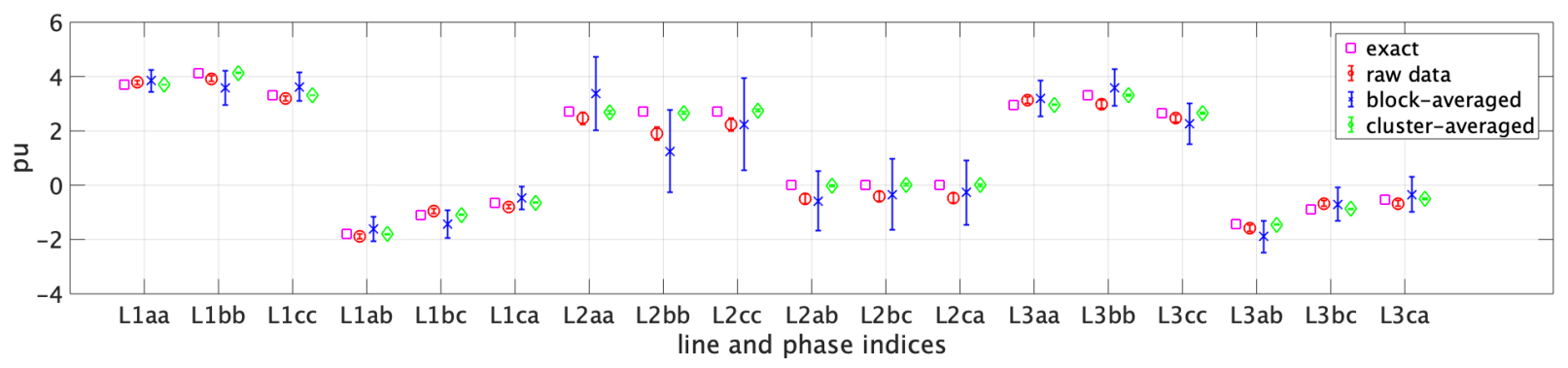

(a) Conductance estimation

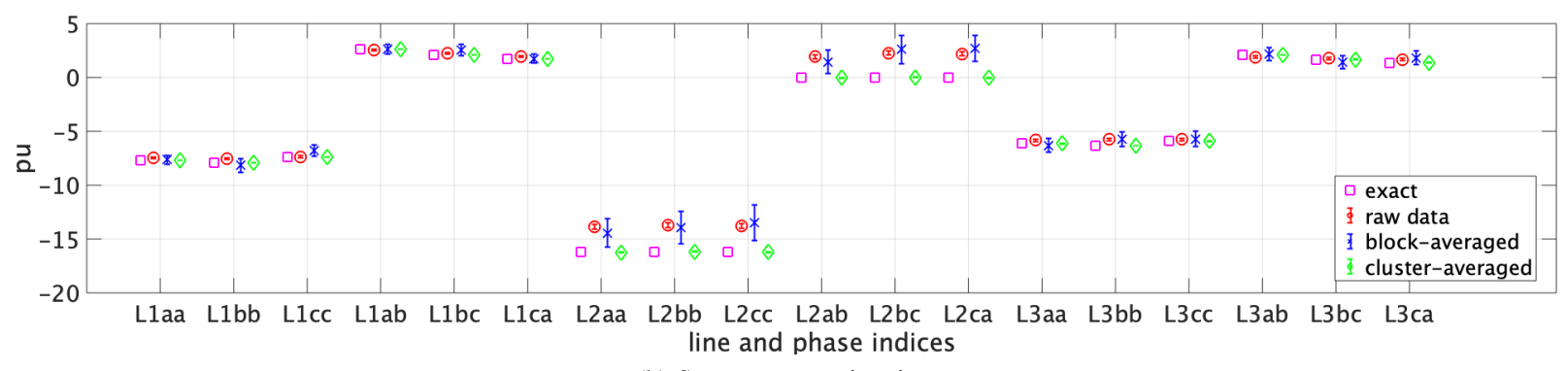

(b) Susceptance estimation

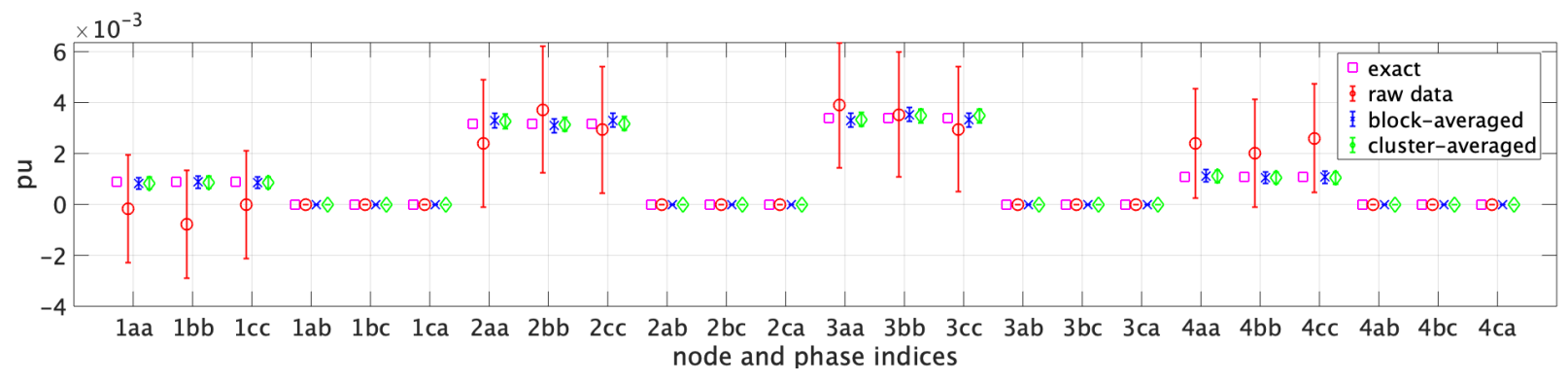

(c) Shunt estimation

Fig. 4. Estimation performance of the parameters (a) conductances (b) susceptance and (c) shunts of the IEEE 4-bus untransposed and unbalanced test network with raw measurements, block-averaged and with cluster-averaged data with LS. Error bars on top of each estimated value shows the uncertainty of the estimates (99\% confidence interval) using (33). L1, L2, L3 refer to different branches and a, b, c refer to different phases of the IEEE 4-bus system in Fig. 3.

are expressed in per unit (pu) as described in Sec. IV-C (we use respectively the base power and voltage of $6 \mathrm{MVA}$ and $4.16 \mathrm{kV}$ corresponding to a base admittance of 0.3467 Siemens.) From the plots, it is clear that the estimation model with clusteraveraging successfully estimates the longitudinal and shunts parameters and has the least uncertainty of the parameter estimates, whereas the estimates using the raw-data and blockaveraging have high biases and uncertainties. Also, it can be observed that the methods using the raw measurements and block-averaging fail to identify the parameters which are zero (line L2 and shunts for all the lines are assumed to have zero off-diagonal elements in the studied IEEE 4-bus test case). Indeed they are estimated to be non-zero, whereas clusteraveraging correctly estimates it.

We also show the heatmaps of the element-wise relative error for the real and imaginary part of the compound admittance matrix. Fig. 5(a-b), (c-d) and (e-f) shows the estimation error for LS with raw-data, block-averaging and cluster-averaging strategies, respectively. The heatmap plots have node indices of the network as $x$ - and $y$ - axes, whereas the color shows the element-wise estimation error on non-zero elements of the admittance matrix using (32). The heatmap has the dimension of 12 on $x$ - and $y$-axis because we are dealing with a 3-phase 4 node system with an admittance matrix dimension of 12 $\times 12$. The comparison shows that the estimations with rawdata and block-averaging have high errors with maximum error upto $200 \%$. In contrast, we obtain near-perfect estimations by using the cluster-averaging strategy with a maximum absolute element-wise error below $3 \%$.

In view of the above, we can conclude that the preprocessing on raw-data by cluster-averaging largely improves the estimation performance compared to using the raw-data directly or simple block-averaging.

1) Other test cases: We also perform estimation on several other networks $(\mathrm{BT}=$ balanced and transposed, $\mathrm{UU}=$ unbalanced and untransposed). The results are summarized in Table III. We show the NMSEs on admittance estimation $\left(\mathcal{E}_{\mathbf{Y}}\right)$ for different publicly available test cases.

From the comparison, it can be seen that the proposed method gives good estimations for all the tested networks. With TLS, we observe that the NMSEs are high in case of rawdata. This happens because the TLS tries to compensate for the 


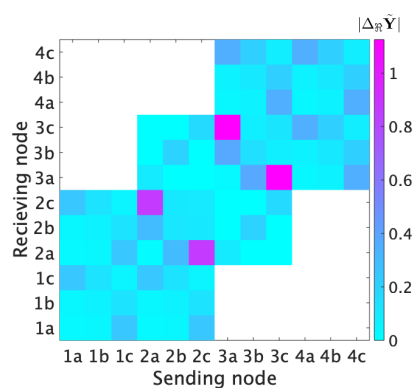

(a)

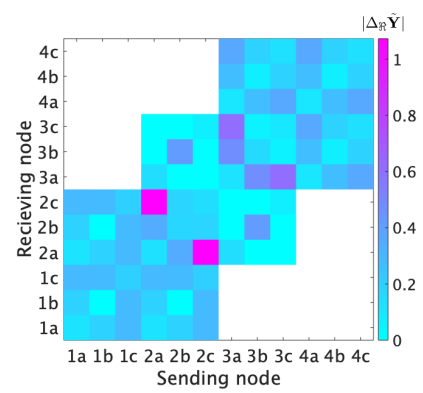

(c)

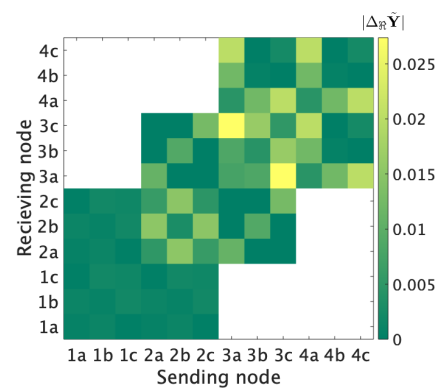

(e)

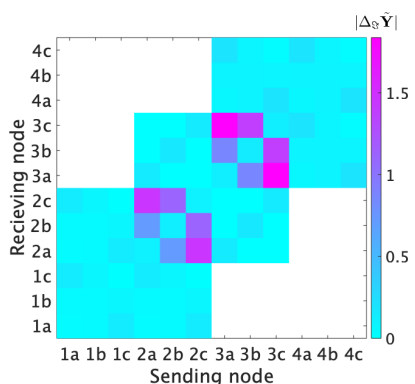

(b)

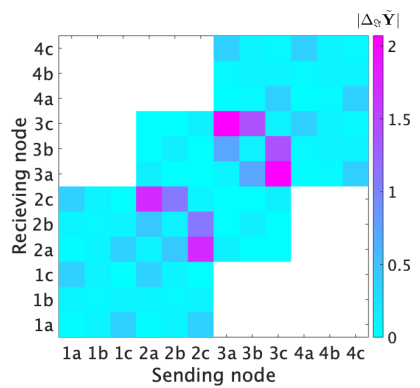

(d)

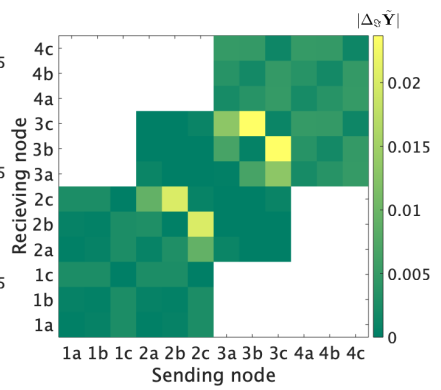

(f)
Fig. 5. Element-wise relative error computed via (32) on real (left) and imaginary (right) part of the estimated compound admittance matrix for the IEEE 4-bus test network using the raw data in (a-b), using the block-averaging in (c-d) and using the cluster-averaged in (e-f) with LS.

TABLE III

NMSE ON ADMITTANCE ESTIMATION FOR DIFFERENT NETWORKS.

\begin{tabular}{|c|c|c|c|c|}
\hline Test-networks & \multicolumn{2}{|c|}{ Raw-data } & \multicolumn{2}{c|}{ Cluster-averaging } \\
\hline & LS & TLS & LS & TLS \\
\hline CIGRE MV (BT) & 0.897 & 6.137 & 0.016 & 0.012 \\
\hline CIGRE LV (BT) & 0.620 & 5.877 & 0.002 & 0.002 \\
\hline CIGRE LV (UU) & 0.371 & 4.77 & 0.033 & 0.037 \\
\hline CIGRE microgrid (BT) & 0.884 & 6.812 & 0.044 & 0.019 \\
\hline IEEE13 (UU) & 0.585 & 2.961 & 0.060 & 0.056 \\
\hline IEEE123 (BT) & 0.946 & 15.551 & 0.060 & 0.060 \\
\hline
\end{tabular}

original measurement noise estimating the true (unobserved) $\widehat{\mathbf{H}}$ and $\widehat{\mathbf{J}}$ along with the admittance parameters resulting in large number of estimation variables compared to the LS. This is mitigated by the cluster-averaging which reduces the noise level on the measurements and number of true measurements to be estimated.

\section{B. Sensitivity analysis}

1) Noise level: we perform estimations using simulated experiments with noise of the IT classes $0.1,0.2,0.5$ and 1 (Table I) according to Algorithm 3. The results are reported for IEEE 4-bus network. We use the same clustering features and $N_{c}$ as in Sec V-A. Table IV shows the NMSEs on admittance estimation for LS and TLS with respect to noise level. As it can be seen, the NMSEs obtained using clusteraveraging is 2-3 order of magnitude better compared to the case without averaging, for all cases. The TLS method with cluster-averaging policy performs the best.

TABLE IV

NMSE ON ADMITTANCE ESTIMATION $\left(\mathcal{E}_{\mathbf{Y}}\right)$ WITH DIFFERENT NOISE LEVELS FOR IEEE 4-BUS NETWORK.

\begin{tabular}{|c|c|c|c|c|}
\hline IT class & \multicolumn{2}{|c|}{ Raw-data } & \multicolumn{2}{c|}{ Cluster-averaging } \\
\hline & LS & TLS & LS & TLS \\
\hline 0.1 & 0.064 & 0.012 & 0.002 & 0.002 \\
\hline 0.2 & 0.181 & 0.038 & 0.003 & 0.003 \\
\hline 0.5 & 0.404 & 0.148 & 0.010 & 0.009 \\
\hline 1 & 0.548 & 9.171 & 0.018 & 0.014 \\
\hline
\end{tabular}

2) Clustering strategy: we investigated different clustering strategies for the cluster-averaging policy. We vary the clustering features used in Algorithm 1 and use them for admittance estimation. The analysis is presented in terms of NMSEs on admittance estimation in Table $\mathrm{V}$ for IEEE 4-bus network with IT class 0.5 . From the analysis, we conclude that the clustering features comprising of the magnitudes of the nodal voltages and currents perform the best.

TABLE V

NMSE ON ADMITTANCE ESTIMATION $\left(\mathcal{E}_{\mathbf{Y}}\right)$ WITH CLUSTERING FEATURES.

\begin{tabular}{|c|c|c|}
\hline Clustering features & LS & TLS \\
\hline Voltage magnitude & 0.036 & 0.024 \\
\hline Current magnitude & 0.021 & 0.021 \\
\hline Voltage and current magnitude & 0.010 & 0.009 \\
\hline Sequence voltage magnitude & 0.066 & 0.432 \\
\hline Sequence current magnitude & 0.054 & 0.056 \\
\hline Sequence voltage and current magnitude & 0.019 & 0.018 \\
\hline
\end{tabular}

We also analysed the sensitivity on the estimation performance with the number of clusters in the cluster-averaging policy. The feature used for this simulation is the dominant feature inferred from the last analysis, namely, the magnitudes of voltages and currents together. The analysis is presented for the IEEE4 system with IT class of 0.5 . We perform cluster averaging on the raw data of 86,400 data points (corresponding to per second single day measurement) with $N_{c}=25,50$, 100, 300, 500, 1000, 5000 and 10,000 (refer to Algorithm 1). The NMSEs on estimated admittance matrix $\left(\mathcal{E}_{\mathbf{Y}}\right)$ are plotted in Fig. 6 for LS and TLS techniques. As it can be observed, the estimation performance improves with decrease in number of clusters. This happens because the clusteraveraging scheme reduces the noise levels on the voltage and current measurements, leading to improvement in estimation quality. The estimations worsen slightly if we go below $N_{c}=100$, this is because the number of input data points 
in the estimation model becomes smaller or comparable to the number of variables to be estimated.

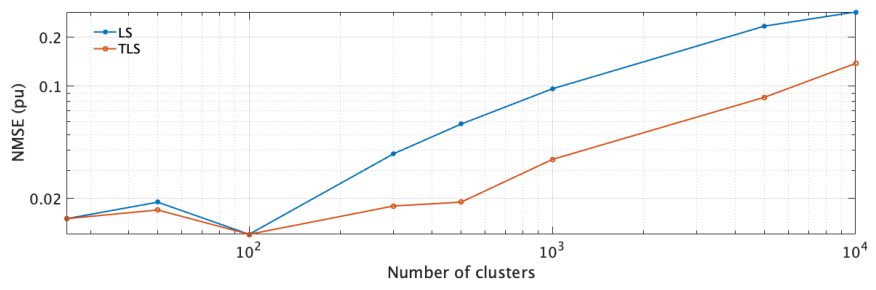

Fig. 6. NMSE $\left(\mathcal{E}_{\mathbf{Y}}\right)$ as a function of the cluster size for IEEE 4-bus network.

\section{Injection current vs branch current model vs both}

We here compare the performance of the proposed estimation process when using: i) injection currents and ii) branch currents along with injection currents. The comparison is done for the IEEE 4-bus system with IT class 0.2 in Table VI. The analysis is presented for LS. We use the same settings for the block- and cluster-averaging as in Sec V-A. The comparison shows that the estimation using the clusteraveraging method is not affected significantly even without branch currents information, whereas the other two methods estimations deteriorate. It is worth noting that the use of the branch current measurements improves the estimation performance significantly, especially for the shunt admittances. This happens because the estimation variables are related to the branch current (as in (14)) individually and can be estimated in a decoupled way, whereas the estimation variables are interlinked to each other in the model relying on injection currents, and so they are not decoupled.

TABLE VI

ESTIMATION PERFORMANCE FOR THE IEEE 4-BUS NETWORK WITH AVAILABILITY OF MEASUREMENTS ON INJECTION CURRENT AND BRANCH CURRENTS.

\begin{tabular}{|c|c|c|c|c|c|}
\hline Data & Current & $\mathcal{E}_{\mathrm{XG}}$ & $\mathcal{E}_{\mathrm{XB}}$ & $\mathcal{E}_{\mathrm{XT}}$ & $\mathcal{E}_{\mathbf{Y}}$ \\
\hline \multirow[b]{2}{*}{ Raw-data } & Injection & 0.138 & 0.213 & 2.392 & 0.221 \\
\hline & $\begin{array}{l}\text { Branch + } \\
\text { Injection }\end{array}$ & 0.124 & 0.172 & 0.337 & 0.181 \\
\hline \multirow{2}{*}{$\begin{array}{c}\text { Block- } \\
\text { averaging } \\
\text { (1-hour block) }\end{array}$} & Injection & 0.381 & 0.201 & 0.101 & 0.239 \\
\hline & $\begin{array}{l}\text { Branch + } \\
\text { Injection }\end{array}$ & 0.191 & 0.145 & 0.016 & 0.163 \\
\hline \multirow{2}{*}{$\begin{array}{c}\text { Cluster- } \\
\text { averaging } \\
\text { (24 clusters) }\end{array}$} & Injection & 0.008 & 0.002 & 0.027 & 0.008 \\
\hline & $\begin{array}{l}\text { Branch + } \\
\text { Injection }\end{array}$ & 0.008 & 0.002 & 0.014 & 0.003 \\
\hline
\end{tabular}

\section{Further Analysis}

In this section, we show how the cluster-averaging method groups the raw-data in different clusters. This analysis gives an insight on why cluster-averaging improves the parameter estimation.

Effect of cluster averaging on the raw measurement data: we show this analysis for the IEEE 4-bus test network (Fig. 3). We use the same clustering features (voltage and current magnitudes) and $N_{c}$ as in Sec V-A.

Fig. 7a shows the distribution of the 1-sec time-indices (86400-time steps) during the day in the 24 clusters as a result of kmeans clustering. In Fig. 7b and Fig. 7c, we also show the variation of the nodal voltage and current magnitudes respectively for the non-zero injection nodes which were used for the clustering. Fig. 7a $y$-axis (left) shows the cluster number, and the corresponding time-steps that fall into clusters are shown in black. In the same plot, we also include the number of elements per cluster on the right $y$-axis of Fig. 7(a). As it can be seen, the clustering does not group the data that is contiguous in time. Instead, data belonging to a given cluster is distributed throughout the day. In particular, the clustering scheme groups measurements with similar magnitudes (considering all the nodes and phases). For example, in cluster- 6 , most of the measurements are balanced among phases with voltage magnitudes close to $1 \mathrm{pu}$ and current injections below 0.05 pu. Similarly, in clusters-9, 19, and 21, the measurements observing a sudden dip in the nodal voltages (caused by increased demand at node 4 phase c) are clustered together. Another example is cluster-12 and 13, which capture slight variation in the voltages due to a dip in demand at node 4 phase a. Clusters-2, 18, and 24 captures sharp generation peaks due to PV injections from node 2 (phase $\mathrm{b}$ ) and node 3 (phase b).

Furthermore, to show the distribution of the data as a result of the averaging strategies, we show zero and negative sequence normalized by positive sequence component for all the nodes for each cluster in the bar plot of Fig. 8. They are defined as follows: Neg. seq. and Zero seq. are defined as the percentage of the negative and zero sequence components with respect to the positive sequence components, respectively. It is given as Neg. seq.

$$
\frac{\left|\mathbf{V}_{\text {neg }}\right|}{\left|\mathbf{V}_{\text {pos }}\right|} \times 100 \%
$$

and Zero seq.

$$
\frac{\left|\mathbf{V}_{\text {zero }}\right|}{\left|\mathbf{V}_{\text {pos }}\right|} \times 100 \%
$$

where $\mathbf{V}_{\text {pos }}, \mathbf{V}_{\text {neg }}, \mathbf{V}_{\text {zero }}$ are positive, negative and zero sequence components respectively.

Fig 8(a) and 8(b) show the barplots corresponding to Neg. seq. and Zero seq., respectively for block-averaged and cluster-averaged data. As seen from the figure, clusteraveraging produces clusters where the positive and negative sequence components are higher than in the block-averaging case. Table VII summarizes the mean and max of Neg.

TABLE VII

Percentage of Negative AND Zero SEQUence Components With RESPECT TO POSITIVE SEQUENCE COMPONENTS.

\begin{tabular}{|c|c|c|c|c|}
\hline Method & \multicolumn{2}{|c|}{ Neg. seq. (\%) } & \multicolumn{2}{c|}{ Zero seq. (\%) } \\
\hline & mean & $\max$ & mean & $\max$ \\
\hline Block-averaging & 0.32 & 0.80 & 0.69 & 2.04 \\
\hline Cluster-averaging & 0.49 & 1.71 & 1.15 & 4.05 \\
\hline
\end{tabular}

seq. and Zero seq. for all the clusters. In view of the above comparisons, it can be seen that the cluster averaging provides higher values of positive and negative sequence components compared to the ones obtained using the block averaging. This feature helps to take into account the contribution of 


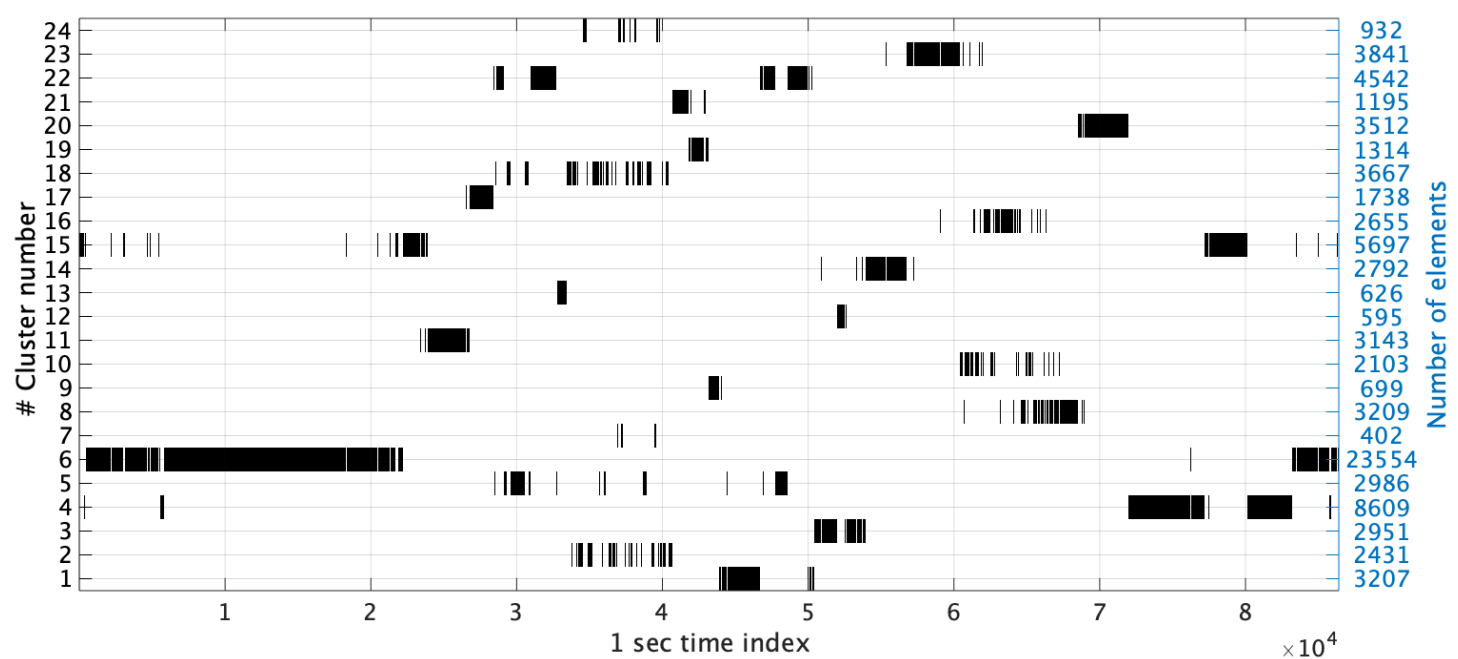

(a)
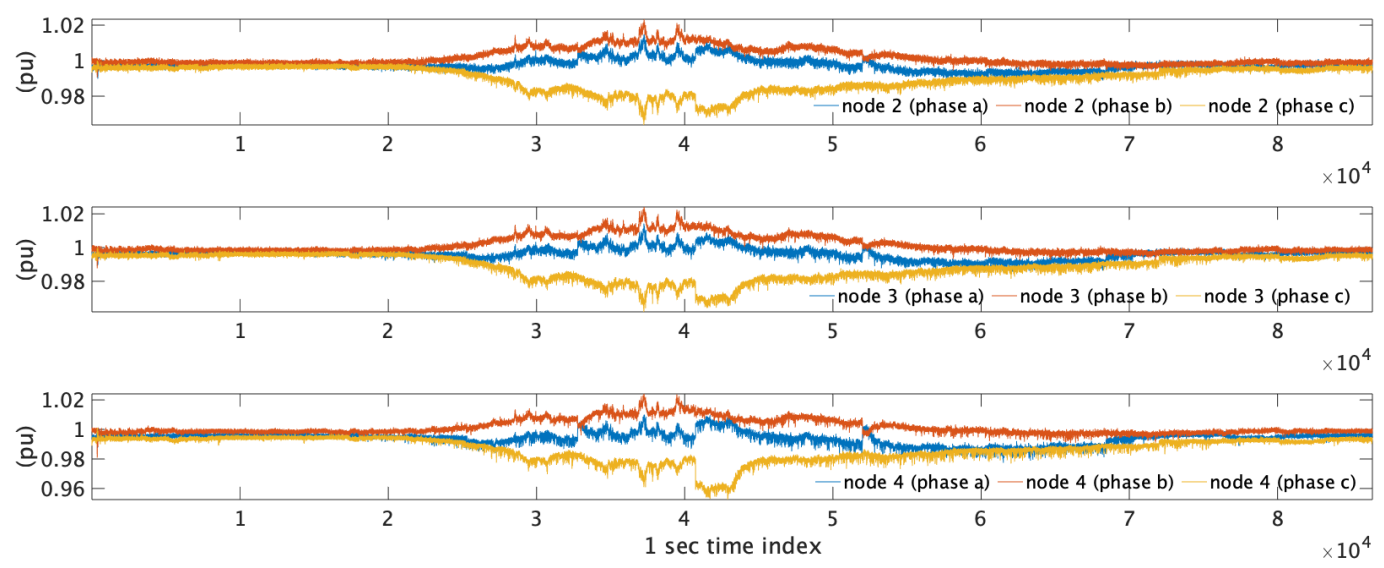

(b)
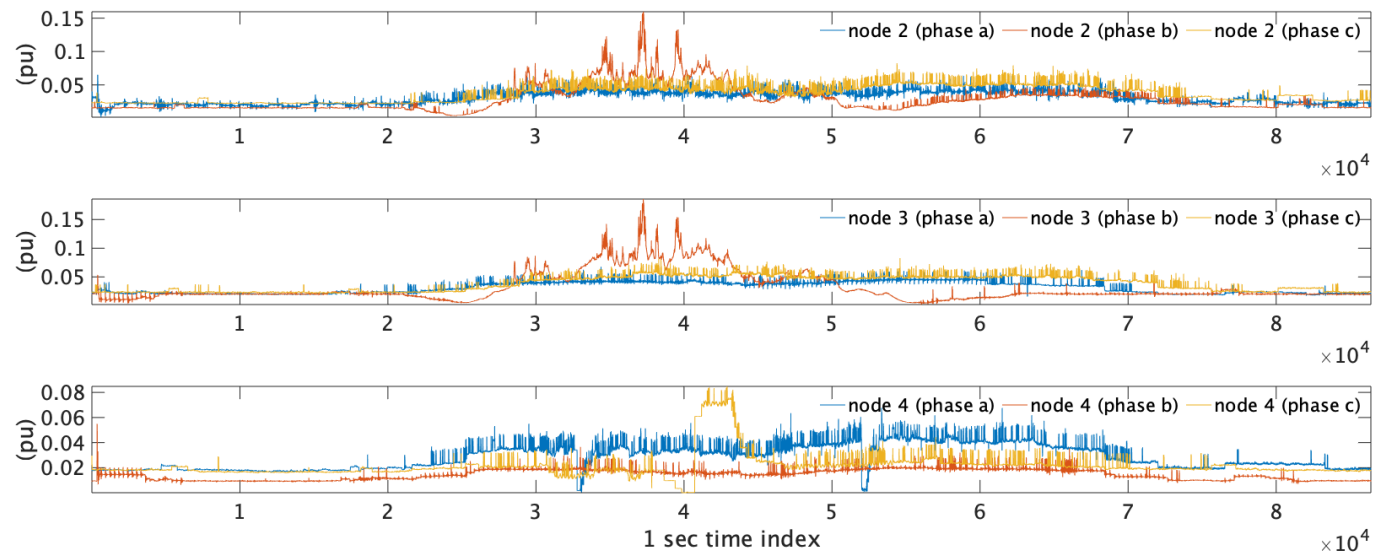

(c)

Fig. 7. Distribution of the 86400 time steps during the day into 24 clusters as a result of cluster averaging: (a) measurements assigned to different clusters with number of elements (right) per cluster, (b) nodal voltage magnitudes (in pu) and (c) nodal current magnitudes (in pu) for all the nodes and phases (except slack node) for $24 \mathrm{hrs}$.

off-diagonal elements of line impedance and shunt admittance matrices in the grid's compound admittance matrix.

\section{CONCLUSIONS AND FUTURE WORKS}

In this paper, we estimate the parameters of compound admittance matrix for generic unbalanced and untransposed three-phase distribution grid. Thanks to the synchrophasor measurements from PMUs, the problem is formulated as a linear estimation model using the measurements of nodal voltages, nodal injection currents and branch currents. We proposed a preprocessing of the measurement data to improve 


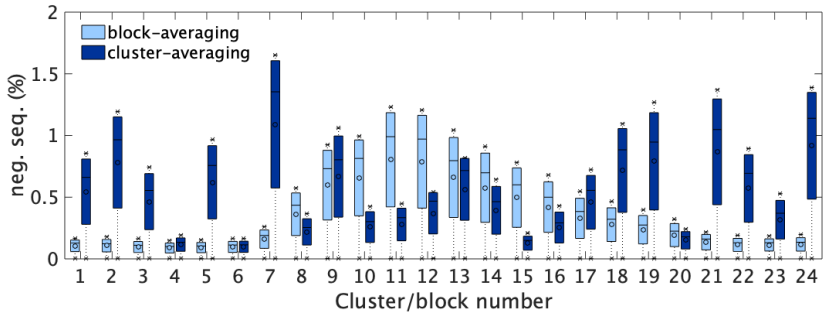

(a)

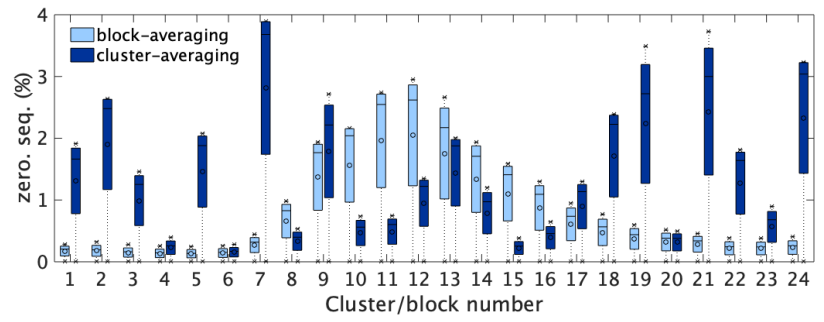

(b)

Fig. 8. Distribution of negative and zero sequence components normalised by the positive sequence component after block-averaging and cluster averaging on raw-data.

the estimation results. The method was validated by simulated experiments on various benchmark networks.

From the analysis, it can be concluded that the proposed method is capable to accurately estimate the longitudinal and shunts elements of a generic unbalanced three-phase system. The pre-processing of data using cluster-averaging improves the estimation performance by two-three order of magnitudes. The sensitivity analysis with noise levels showed that the preprocessing method works even with low accuracy class of IT (i.e. class 1). The estimations with cluster-averaged data performs best when magnitudes of the nodal voltages and currents are used as features in the clustering scheme. The estimation performs better with fewer clusters. It was also found that the information on branch currents improves the estimation performance, especially on the networks with nonnegligible shunt elements.

Possible extensions of this work include investigating the estimation problem considering biased measurements from non-calibrated IT, fewer PMUs, and the impact of timevarying environmental factors such as conductor temperature and line thermal loading to estimate the varying nature of grid parameters (i.e. line resistances).

\section{REFERENCES}

[1] P. Kundur, N. Balu, and M. Lauby, Power system stability and control. McGraw-Hill, 1994.

[2] F. Milano, Power system modelling and scripting. Springer Science \& Business Media, 2010.

[3] A. Abur and A. G. Exposito, Power system state estimation: theory and implementation. CRC press, 2004.

[4] P. Mercier, R. Cherkaoui, and A. Oudalov, "Optimizing a battery energy storage system for frequency control application in an isolated power system," IEEE Trans. Power Syst., vol. 24, no. 3, pp. 1469-1477, 2009.

[5] G. Celli, E. Ghiani, S. Mocci, and F. Pilo, "A multiobjective evolutionary algorithm for the sizing and siting of distributed generation," IEEE Transactions on power systems, vol. 20, no. 2, pp. 750-757, 2005.

[6] F. C. Schweppe and E. J. Handschin, "Static state estimation in electric power systems," Proc. of the IEEE, vol. 62, no. 7, pp. 972-982, 1974.
[7] M. Pignati, L. Zanni, P. Romano, R. Cherkaoui, and M. Paolone, "Fault detection and faulted line identification in active distribution networks using synchrophasors-based real-time state estimation," IEEE Transactions on Power Delivery, vol. 32, no. 1, pp. 381-392, 2016.

[8] L. G. Perez and A. J. Urdaneta, "Optimal computation of distance relays second zone timing in a mixed protection scheme with directional overcurrent relays," IEEE Trans. Power Del., vol. 16, pp. 385-388, 2001.

[9] W. H. Kersting, Distribution system modeling and analysis. CRC press, 2012.

[10] A. Egozi, "Subscriber line impedance measurement device and method," Nov. 7 1995, uS Patent 5,465,287.

[11] M. Pau, P. A. Pegoraro, and S. Sulis, "WLS distribution system state estimator based on voltages or branch-currents: Accuracy and performance comparison," in I2MTC. IEEE, 2013, pp. 493-498.

[12] G. D'Antona, "Uncertainty of power system state estimates due to measurements and network parameter uncertainty," in International Workshop AMPS. IEEE, 2010, pp. 37-40.

[13] K. Christakou, M. Paolone, and A. Abur, "Voltage control in active distribution networks under uncertainty in the system model: a robust optimization approach," IEEE Transactions on Smart Grid, vol. 9, no. 6 pp. 5631-5642, 2017.

[14] G. Sivanagaraju, S. Chakrabarti, and S. C. Srivastava, "Uncertainty in transmission line parameters: Estimation and impact on line current differential protection," IEEE Transactions on Instrumentation and Measurement, vol. 63, no. 6, pp. 1496-1504, 2013.

[15] NASPI Distribution Task Team, "Synchrophasor monitoring for distribution systems technical foundations and applications [white paper]," 2018.

[16] L. Reyes-Chamorro, A. Bernstein, J.-Y. L. Boudec, and M. Paolone, "A composable method for real-time control of active distribution networks with explicit power setpoints. part ii: Implementation and validation," Electric Power Systems Research, 2015.

[17] R. K. Gupta, F. Sossan, and M. Paolone, "Grid-aware distributed model predictive control of heterogeneous resources in a distribution network: Theory and experimental validation," IEEE Transactions on Energy Conversion, 2020

[18] R. P. Schulz, L. S. VanSlyck, and S. H. Horowitz, "Applications of fast phasor measurements on utility systems," in Conference Papers Power Industry Computer Application Conference. IEEE, 1989, pp. 49-55.

[19] I. W. Slutsker, S. Mokhtari, and K. A. Clements, "Real time recursive parameter estimation in energy management systems," IEEE Transactions on power systems, vol. 11, no. 3, pp. 1393-1399, 1996.

[20] I.-D. Kim and R. K. Aggarwal, "A study on the on-line measurement of transmission line impedances for improved relaying protection," Int. J. Elect. Power \& Energy Syst., vol. 28, no. 6, pp. 359-366, 2006.

[21] C. Indulkar and K. Ramalingam, "Estimation of transmission line parameters from measurements," International Journal of Electrical Power \& Energy Systems, vol. 30, no. 5, pp. 337-342, 2008.

[22] D. Shi, D. J. Tylavsky, K. M. Koellner, N. Logic, and D. E. Wheeler, "Transmission line parameter identification using pmu measurements," European Transactions on Electrical Power, vol. 21, no. 4, pp. 15741588, 2011.

[23] L. Ding, T. Bi, and D. Zhang, "Transmission line parameters identification based on moving-window tls and pmu data," in Int. Conf. APAP, vol. 3. IEEE, 2011, pp. 2187-2191.

[24] H. Zhang, Z. Diao, and Y. Cui, "Identification of Power Network Branch Parameters Based on State Space Transformation," IEEE Access, vol. 7, pp. 91720-91730, 2019.

[25] Y. Yuan, O. Ardakanian, S. Low, and C. Tomlin, "On the inverse power flow problem," arXiv preprint arXiv:1610.06631, 2016.

[26] J. Yu, Y. Weng, and R. Rajagopal, "Patopa: A data-driven parameter and topology joint estimation framework in distribution grids," IEEE Transactions on Power Systems, vol. 33, no. 4, pp. 4335-4347, 2018.

[27] P. A. Pegoraro, K. Brady, P. Castello, C. Muscas, and A. von Meier, "Line impedance estimation based on synchrophasor measurements for power distribution systems," IEEE Transactions on Instrumentation and Measurement, vol. 68, no. 4, pp. 1002-1013, 2018.

[28] P. A. Pegoraro et al., "Compensation of systematic measurement errors in a pmu-based monitoring system for electric distribution grids," IEEE Transactions on Instrumentation and Measurement, vol. 68, no. 10, pp. 3871-3882, 2019.

[29] H. Goklani, G. Gajjar, and S. Soman, "Instrument transformer calibration and robust estimation of transmission line parameters using pmu measurements," IEEE Transactions on Power Systems, 2020.

[30] C. Wang, V. A. Centeno, K. D. Jones, and D. Yang, "Transmission lines positive sequence parameters estimation and instrument transformers 
calibration based on pmu measurement error model," IEEE Access, vol. 7, pp. 145 104-145 117, 2019.

[31] G. H. Golub and C. F. Van Loan, "An analysis of the total least squares problem," SIAM journal on numerical analysis, vol. 17, no. 6, pp. 883$893,1980$.

[32] C. T. F. C6.04.02, "Benchmark systems for network integration of renewable and distributed energy resources," Cigre' International Council on large electric systems, Tech. Rep., July 2009.

[33] W. H. Kersting, "Radial distribution test feeders," in 2001 IEEE Power Engineering Society Winter Meeting. Conference Proceedings, vol. 2. IEEE, 2001, pp. 908-912.

[34] V. Milojević, S. Calija, G. Rietveld, M. V. Ačanski, and D. Colangelo, "Utilization of pmu measurements for three-phase line parameter estimation in power systems," IEEE Transactions on Instrumentation and Measurement, vol. 67, no. 10, pp. 2453-2462, 2018.

[35] P. K. Mansani, A. Pal, M. Rhodes, and B. Keel, "Estimation of transmission line sequence impedances using real pmu data," in 2018 North American Power Symposium (NAPS). IEEE, 2018, pp. 1-6.

[36] R. S. Singh, S. Babaev, V. Cuk, S. Cobben, and H. van den Brom, "Line parameters estimation in presence of uncalibrated instrument transformers," in 2019 2nd International Colloquium on Smart Grid Metrology (SMAGRIMET). IEEE, 2019, pp. 1-8

[37] A. Wehenkel, A. Mukhopadhyay, J.-Y. Le Boudec, and M. Paolone, "Parameter estimation of three-phase untransposed short transmission lines from synchrophasor measurements," IEEE Transactions on Instrumentation and Measurement, vol. 69, no. 9, pp. 6143-6154, 2020.

[38] A. M. Kettner and M. Paolone, "On the properties of the compound nodal admittance matrix of polyphase power systems," IEEE Transactions on Power Systems, vol. 34, no. 1, pp. 444-453, 2018.

[39] S. Van Huffel and P. Lemmerling, Total least squares and errors-invariables modeling: analysis, algorithms and applications. Springer Science \& Business Media, 2013.

[40] I. Markovsky and S. Van Huffel, "Overview of total least-squares methods," Signal processing, vol. 87, no. 10, pp. 2283-2302, 2007.

[41] D. Arthur and S. Vassilvitskii, "k-means++: The advantages of careful seeding," Stanford, Tech. Rep., 2006.

[42] Instrument Transformers:, "Additional requirements for current transformers," Standard IEC, pp. 61 869-2, 2014.

[43] Instrument Transformers:, "Additional requirements for voltage transformers," Standard IEC, pp. 61 869-3, 2011.

[44] M. Pignati et al., "Real-time state estimation of the epfl-campus medium-voltage grid by using pmus," in Conf. Innovative Smart Grid Technologies (ISGT), no. 1-5, 2015.

[45] P. Romano and M. Paolone, "Enhanced interpolated-dft for synchrophasor estimation in fpgas: Theory, implementation, and validation of a pmu prototype," IEEE Transactions on Instrumentation and Measurement, vol. 63, no. 12, pp. 2824-2836, 2014.

[46] M. Paolone, J.-Y. Le Boudec, S. Sarri, and L. Zanni, "Static and recursive pmu-based state estimation processes for transmission and distribution power grids," IET, Tech. Rep., 2015.

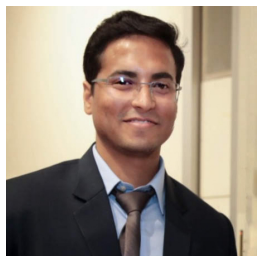

Rahul Kumar Gupta completed his B.Tech in electrical engineering at NIT Rourkela, India in 2014. From 2015 to 2016, he worked as research assistant on micro-energy harvesting at NUS Singapore. In 2018, he received his M.Sc degree in electrical engineering with orientation in smart grids technology at EPFL Lausanne, Switzerland. He received Zanelli: technologie et développement durable prize 2018 for his master project in the field of sustainable development. Currently, he is pursuing his Ph.D. degree at the Distributed Electrical Systems Laboratory, EPFL. His research interests include model predictive control, distributed optimization and data-driven control of the active distribution networks in the presence of uncertainties.

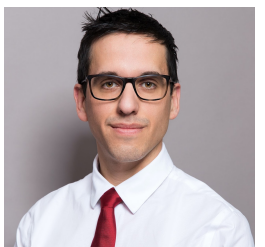

Fabrizio Sossan completed his studies in computer engineering at the University of Genova in 2010, and he obtained his Ph.D. in Electrical Engineering from the Technical University of Denmark (DTU) in 2014. From 2014 to 2017, he was a postdoctoral fellow at EPFL Lausanne in Switzerland, in 2018 a guest scientist at NREL in the USA and a scientist at ETH in Switzerland. From 2019, he is a senior researcher and lecturer at MINES ParisTech in France. His research interests are modeling and operation of distributed energy resources and energy storage, including forecasting, for renewable power grids. He is an associate editor of Elsevier Sustainable Energy Grids and Networks.

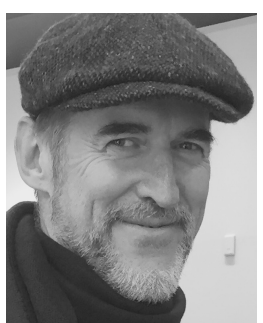

Jean-Yves Le Boudec is professor at EPFL and fellow of the IEEE. He graduated from Ecole Normale Supérieure de Saint-Cloud, Paris, where he obtained the Agrégation in Mathematics in 1980 and received his doctorate in 1984 from the University of Rennes, France. From 1984 to 1987 he was with INSA/IRISA, Rennes. In 1987 he joined Bell Northern Research, Ottawa, Canada, as a member of scientific staff in the Network and Product Traffic Design Department. In 1988, he joined the IBM Zurich Research Laboratory where he was manager of the Customer Premises Network Department. In 1994 he became associate professor at EPFL. His interests are in the performance and architecture of communication systems and smart grids. He co-authored a book on network calculus, which serves as a foundation for deterministic networking, an introductory textbook on Information Sciences, and is the author of the book "Performance Evaluation".

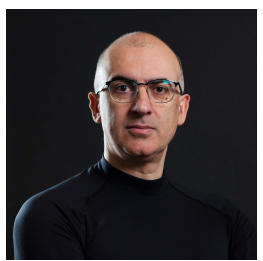

Mario Paolone (M'07-SM'10) received the M.Sc. (Hons.) and Ph.D. degrees in electrical engineering from the University of Bologna, Italy, in 1998 and 2002. In 2005, he was an Assistant Professor in power systems with the University of Bologna, where he was with the Power Systems Laboratory until 2011. Since 2011, he has been with the Swiss Federal Institute of Technology, Lausanne, Switzerland, where he is currently Full Professor and the Chair of the Distributed Electrical Systems Laboratory. His research interests focus on power systems with particular reference to real-time monitoring and operation aspects, power system protections, dynamics and transients. Dr. Paolone has authored or coauthored over 300 papers published in mainstream journals and international conferences in the area of energy and power systems that received numerous awards including the 2013 IEEE EMC Technical Achievement Award, two IEEE Transactions on EMC best paper awards and the Basil Papadias best paper award at the 2013 IEEE PowerTech. Dr. Paolone was the founder Editorin-Chief of the Elsevier journal Sustainable Energy, Grids and Networks. 